\title{
Les théories modernes du magnétisme et leurs applications
}

\author{
par Louis NEEL $\left(^{*}\right)$
}

\section{INTRODUCTION}

Comme toutes les théories relatives aux propriétés physiques de l'état solide, la théorie des propriétés magnétiques des métaux s'est développée très lentement, et nos connaissances sur le mécanisme de l'aimantation du fer n'ont guère commencé à se préciser que depuis une quinzaine d'années. D'ailleurs, ce qui complique les choses, les applications industrielles importantes sont plutôt liées aux défauts accidentels de la structure cristalline qu'à cette structure elle-même et aux propriétés atomiques : par exemple, pour le même diagramme de rayons $\mathrm{X}$, le champ coercitif du fer peut varier entre 1.000 gauss et $1 / 1.000$ de gauss.

Pour limiter notre sujet, nous nous propocons d'examiner dans quelle mesure les progrès théoriques récents relatifs au ferromagnétisme ont apporté, ou sont susceptibles d'apporter, une aide dans le domaine des applications.

\section{CONDITIONS DU FERROMAGNÉTISME}

Pour qu'une substance soit ferromagnétique, il faut réunir deux conditions : il faut d'abord que les atomes possèdent un moment magnétique et ensuite qu'entre ces atomes existent des forces suffisantes pour amener les moments magnétiques élémentaires à être parallèles entre eux malgré l'action désorganisatrice de l'agitation thermique : c'est en effet grâce à ce parallélisme que l'aimantation de la substance peut devenir sensible du point de vue macroscopique.

Pour que l'atome possède un moment magnétique, il faut qu'en dehors des électrons de liaison et des électrons de conduction, existe une couche électronique interne in-

(*) Conférence faite à la séance de clôture des Journées Métallurgiques de la Société Française de Métallurgie, le 10 octobre 1947. complète, dite couche magnétique, comme cela se produit dans les différentes séries de transition, en particulier la première qui comprend notamment le nickel, le cobalt, le fer et le manganèse, ou dans la série des terres rares. Il faut, d'ailleurs, noter que le moment magnétique d'un atome, un atome de fer par exemple, n'est pas une propriété spécifique de cet atome, car la couche électronique d'où provient le moment est soumise aux influences perturbatrices des atomes voisins; dans beaucoup de métaux, par exemple le nickel, on est amené à concevoir une sorte d'équilibre électronique entre la couche magnétique et la couche des électrons de conduction. C'est un problème difficile, bien loin encore d'être résolu, de sorte qu'il n'est pas possible aujourd'hui de prédire avec précision la valeur d'un moment atomique à l'exception de quelques cas simples comme celui du gadolinium dans lequel la couche magnétique est suffisamment profonde pour que les perturbations extérieures soient négligeables : la théorie et l'expérience donnent 7 magnétons de Bohr. 


\section{LES FORCES DE HEISENBERG}

Les forces les plus intenses qui existent entre les moments magnétiques des atomes, sont, comme l'a montré Heisenberg, d'origine électrostatique; ce sont des actions d'échange entre électrons analogues aux forces qui produisent la liaison chimique homopolaire. Elles peuvent être négatives et tendre à aligner en sens inverse les moments magnétiques de deux atomes voisins, comme cela se produit pour le manganèse et le chrome qui possèdent un faible paramagnétisme indépendant de la température. On dit alors qu'il y a antiferromagnétisme. Elles peuvent être positives, et on obtient alors du ferromagnétisme. Actuellement, la théorie n'est pas encore capable de prédire le signe de ces forces ni, a fortiori, leur intensité. On sait seulement qu'elles doivent décroître très rapidement avec la distance de manière à être complètement négligeables à une distance de 4 ou 5 angströms. Ce sont des forces qui ne peuvent assurer le parallélisme qu'entre les moments élémentaires d'atomes peu éloignés les uns des autres : en un mot, elles assurent l'ordre à petite distance.

Si ces forces existaient seules, rien n'empêcherait la direction des moments atomiques de tourner progressivement, par rapport à la direction des axes cristallins, d'un point à un autre, de la substance, pourvu que la variation de direction entre deux atomes proches voisins soit suffisamment faible. Les propriétés magnétiques d'une telle substance seraient extrêmement simples, car il suffirait alors d'un champ magnétique infiniment faible pour rendre parallèles entre elles les directions des aimants élémentaires dans l'ensemble de la substance, c'est-à-dire pour produire l'aimantation à saturation. A part certains ferro-nickels, de composition bien spéciale et extrêmement purs, qui se comportent effectivement de cette manière, on sait que les propriétés magnétiques des ferromagnétiques réels sont bien différentes.

\section{LES FORCES MAGNÉTO-CRISTALLINES}

En réalité, il existe d'autres forces qui jouent un rôle extrêmement important bien que beaucoup plus faibles que les forces de Heisenberg, mille fois moins pour fixer les idées : ce sont les forces magnéto-cristallines qui assurent un certain couplage entre la direction des moments atomiques et la direction des axes cristallins. Elles proviennent de la déformation des atomes dans le réseau cristallin; leur théorie est difficile et il n'est guère possible que d'estimer leur ordre de grandeur; mais pour en étudier les effets, il suffit de savoir que l'énergie qui leur est liée est minimum lorsque les moments atomiques snnt orientés suivant certaines directions du réseau cristallin appelées directions privilégiées ou directions de facile aimantation, puisque ce sont les directions que prendraient spontanément les moments élémentaires en l'absence de forces perturbatrices. Dans le fer, ces directions privilégiées sont les axes quaternaires, et il faut exercer un champ magnétique de plusieurs centaines de gauss pour en écarter les moments élémentaires.

Il en résulte que dans un morceau de fer polycristallin, même extrêmement pur, il faut exercer un champ magnétique notable, de plusieurs centaines de gauss, pour produire l'aimantation à saturation, puisqu'il faut pour cela écarter de la direction de facile aimantation les moments magnétiques élémentaires des grains qui sont orientés de manière défavorable. C'est pour cette raison que les tôles de dynamo ou de transformateur ne peuvent pratiquement travailler qu'à des aimantations qui ne dépassent guère la moitié de la saturation, au plus les deux tiers. On a pu améliorer cet état de choses par des traitements physiques destinés à orienter les grains élémentaires, en rapprochant la direction de leurs axes quaternaires de la direction d'aimantation. On obtient ainsi les alliages connus sous le nom d'hypersils qui ont été étudiés et mis au point en Amérique.

\section{LES DOMAINES ÉLÉMENTAIRES}

Du point de vue du mécanisme de l'aimantation, ces forces magnétocristallines jouent un rôle important en assurant cet ordre à longue distance que les forces de Heisenberg ne sont pas capables de produire malgré leur plus grande intensité. Dans ces conditions, pourvu que le champ magnétique auquel est soumise une substance ferromagnétique ne dépasse pas quelques dizaines de gaus:, elle se fragmentera en domaities à l'intérieur desquels les aimants élémentaires seront tous parallèles à la même direction privilégiée, ce sont les domaines élémentaires de Weiss : leur dimension est fort variable, de l'ordre de quelques microns. 


\section{LES DIFFÉRENTS MÉCANISMES DE L'AIMANTATION}

L'aimantation macroscopique est la résultante géométrique des aimantations des différents domaines élémentaires suivant lesquels la substance est subdivisée et qui ont des directions différentes puisqu'il existe plusieurs directions de facile aimantation énergétiquement équivalentes. On peut alors concevoir deux façons différentes de changer la valeur de l'aimantation macroscopique. On peut faire tourner en bloc l'aimantation dans chaque domaine, mais il faut pour cela surmonter les forces magnétocristallines et disposer de champs magnétiques importants, de plusieurs centaines de gauss dans le cas du fer; c'est donc un processus difficile : c'est le mécanisme de la rotation. On peut aussi déplacer progressivement les frontières de séparation entre les domaines élémentaires de manière à augmenter, aux dépens des autres domaines, les dimensions des domaines dont les moments élémentaires sont orientés d'une manière favorable par rapport à la direction d'aimantation choisie. En principe, ce processus par déplacement de paroi ne demande pas d'énergie, tout au moins dans une subtance pure; il est donc uniquement responsable des variations d'aimantation qui sont à la base des applications industrielles des alliages magnétiquement doux.

\section{NATURE DES PAROIS DE SÉPARATION ENTRE LES DONAINES ÉLÉMENTAIRES}

Pour préciser la théorie du phénomène, il importe d'abord d'élucider la nature de ces frontières de séparation entre les domaines élémentaires, ou plus simplement de ces parois. Elles ne sauraient être infiniment minces puisqu'il y aurait alors brusque variation de la direction des moments élémentaires contraire à l'ordre à petite distance imposé par les forces de Heisenberg. L'aimantation doit donc changer progressivement de direction, ce qui impose une certaine épaisseur à la paroi, mais cette épaisseur ne doit pas être trop grande à cause de l'énergie à dépenser, contre les forces magnétocristallines, pour écarter, à l'intérieur de la paroi, les moments élémentaires des directions privilégiées. Un compromis s'établit entre ces deux tendances opposées, dont la théorie a été établie par Bloch. La paroi prend une épaisseur finie qui pour le fer est de l'ordre du dixième de micron, tandis que l'énergie à dépenser pour créer la paroi est de l'ordre de l'erg par centimètre carré. Cette énergie de paroi peut aussi être considérée comme une tension superficielle.

\section{LES SUBSTANCES FERROMAGNÉTIQUES FINEMENT SUBDIVISÉES}

Il résulte immédiatement de ces données que ce facile processus de variation d'aimantation par déplacement de paroi ne peut se produire que s'il existe des parois, c'està-dire si les dimensions de la substance sont suffisantes pour loger ces parois. On prévoit ainsi qu'il ne pourra pas exister de parois dans les grains d'une poudre suffisamment fine, de quelques centaines d'angströms par exemple, de sorte que les variations d'aimantation ne pourront alors provenir que du difficile processus de rotation dont nous avons parlé plus haut. Une fois qu'une telle poudre aura été aimantée, il sera difficile d'en faire varier l'aimantation : en d'autres termes, elle pourra constituer un aimant permanent. L'expérience justifie pleinement ces prévisions : en effet, nous avons pu, à Grenoble, avec la collaboration de MM. Aubry et Weil, en amenant du fer, par des méthodes appropriées, à l'état de grains de quelques centaines d'angströms de diamètre, lui communiquer un champ coercitif supérieur à millo gauss. D'ailleurs dans de telles poudres, ce ne sont plus les forces magnétocristallines qui maintiennent l'aimantation suivant certaines directions privilégiées mais des forces purement magnétiques liées à la forme du grain : le grain est toujours inévitablement de forme irrégulière et on peut montrer, qu'à cause des effets du champ démagnétisant, l'aimantation tend à s'orienter dans le sens de la plus grande dimension. Cet effet dépasse de beaucoup, dans le cas du fer, celui des forces magnétocristallines. En comprimant les poudres obtenues, on obtient des aimants industriellement utilisables, dont le champ coercitif est un peu inférieur à celui de la poudre non comprimée à cause de la diminution des champs démagnétisants provoqués par l'agglomération. 


\section{LES OBSTACLES AU DÉPLACEMENT DES PAROIS}

Si nous revenons maintenant aux substances compactes, dans lesquelles les variations d'aimantation proviennent des déplacements des parois, il apparaît que les parois se déplacent librement, sans dépense d'énergie, dans les substances idéa'ement pures; leur champ coercitif doit donc être nul. Il s'agit donc d'examiner maintenant la nature des obstacles qui, dans une substance réelle, s'opposent à leur libre déplacement. A priori, il est évident que si la substance n'est pas homogène, toutes ses propriétés, et en particulier l'énergie de paroi, varieront d'un point à l'autre. Il existera donc certaines positions de la paroi pour lesquelles l'énergie sera minimum : il faudra donc exercer un champ magnétique fini pour l'en écarter, d'où une cause d'hystérésis.

Les premiers défauts d'homogénéité qui, à cet égard, aient attiré l'attention et fait l'objet des théories de Becker et de ses collaborateurs, sont les tensions internes : ces tensions internes altèrent localement la symétrie du réseau et modifient la valeur de l'énergie magnétocristalline qui se trouve être extrêmement sensible aux variations dans la disposition géométrique des atomes autour d'un atome central donné. Ce phénomène est d'ailleurs étroitement lié à la magnétostriction, car, inversement, toutes les modifications d'aimantation sont accompagnées de modifications dans la disposition et la distance des atomes voisins. Il en résulte que dans un corps à magnétostriction nulle l'effet des tensions internes sur l'aimantation est également nul.

Un autre défaut d'homogénéité, dont le rôle a d'abord été signalé par Kersten, est constitué par la présence de cavités ou d'inclusions non magnétiques ou moins magnétiques que la phase principale. Lorsque la paroi bissecte une cavité, la portion correspondante de paroi disparaît, et il y a diminution d'énergie : la paroi est en quelque sorte retenue par la cavité.
En somme, d'après ces théories, les phénomènes d'hystérésis proviendraient des variations de la tension superficielle des parois de séparation entre les domaines élémentaires, variations liées aux défauts internes d'homogénéité de la substance.

Un examen plus attentif montre que ce phénomène ne suffit pas à rendre compte des valeurs élevées de l'hystérésis présentées par les bons aimants permanents modernes. Des travaux théoriques effectués dans notre laboratoire, il ressort que les défauts d'homogénéité agissent en réalité en déviant les moments magnétiques élémentaires de leur direction idéale. Au lieu d'être tous parallèles, comme dans un domaine élémentaire d'un cristal idéal, ils sont tous plus ou moins irrégulièrement déviés autour d'une direction moyenne. Comme l'aimantation n'est plus rigoureusement uniforme, des charges magnétiques fictives apparaissent à l'intérieur de la substance; cette densité de charge, $0=-$ div I, varie irrégulièrement d'un point à l'autre, tantôt négative, tantôt positive. Ces densités de charges changent d'ailleurs de signe, en conservant les mêmes valeurs absolues, lorsqu'on retourne l'aimantation bout pour bout. Supposons alors qu'une paroi séparant deux domaines, à l'intérieur desquels les aimantations sont dirigées en sens inverses, vienne bissecter une région $\mathrm{R}$ où existait primitivement une forte densité positive de charges magnétiques. A mesure que la paroi traverse $\mathrm{R}$, les charges positives deviennent négatives, si bien que lorsque la paroi est au milieu de $\mathrm{R}$, des charges positives se trouvent être voisines de charges négatives, d'où diminution de l'énergie. Il faudra dépenser du travail pour écarter la paroi de cette position. Ce mécanisme d'hystérésis est nettement plus important que celui des variations de tension superficielle de paroi.

\section{ROLE DE L'ÉCHELLE DES DÉFAUTS}

Ce mécanisme permet aussi de nous rendre compte que les défauts d'homogénéité, pour être efficaces du point de vue de l'hystérésis, ne doivent pas être de dimensions trop faibles : leur échelle ne doit pas être trop petite. En effet, si cette échelle était trop petite, les forces de Heisenberg qui sont de puissantes forces d'ordre à courte distance, empêcheraient l'aimantation de dévier d'une façon appréciable de sa direction initiale, de sorte que le mécanisme étudié ne jouerait plus. Il faut donc que l'échelle des défauts soit suffisante : de l'ordre du dixième de micron dans le fer, comme une étude mathématique précise du phénomène permet de le montrer. Des inégalités de concentration à l'échelle atomique, comme il peut s'en produire dans les alliages, ne sauraient ainsi provoquer d'hystérésis. Remarquons, en passant que ces défauts à l'échelle atomique peuvent être mis en évidence et étudiés par les déplacements du point de Curie et les changements dans la forme de la courbe d'aimantation au voisinage de ce point.

Inversement, si l'échelle des défauts est trop grande, comme ce serait le cas pour des trous de $1 \mathrm{~mm}$ de diamètre dans un morceau de fer, il ne se produit plus de simples déviations irrégulières de l'aimantation, à l'intérieur d'un domaine, autour d'une direction moyenne, mais plutôt une réorganisation complète de la distribution des domaines élémentaires pour laquelle les déviations sont à nouveau faibles. Le mécanisme étudié plus haut ne joue pas non plus. Ainsi, des défauts d'homogénéité d'échelle supérieure à une certaine limite, qui est de l'ordre de 100 à 1.000 microns, ne provoquent pas d'hystérésis dans les ferromagnétiques. A l'extrême limite, on sait qu'une déformation homogène, comme la tension d'un fil au-dessous de sa limite élastique n'augmente pas le champ coercitif. 


\section{INFLUENCE DES DÉFAUTS A GRANDE ÉCHELLE SUR L'APPROCHE A LA SATURATION}

Il faut bien préciser, cependant, que les défauts à grande échelle, sans influer sensiblement sur l'hystérésis, modifient d'une manière considérable le reste de la courbe d'aimantation, notamment la forme de la loi d'approche à la saturation dans les champs magnétiques élevéz. Plu; il $\mathrm{y}$ a de défauts et plus il devient difficile d'aimanter à saturation. C'est ainsi qu'un morceau de fer obtenu par frittage et présentant une très grande porosité, caractérisée par une densité de 5,5 par exemple, est plus dur, magnétiquement, que le cobalt : dans 10.000 gauss, la saturation est loin d'être atteinte, bien que mécaniquement la substance puisse être extrêmement douce.

\section{DIFFÉRENCIATION MAGNÉTIQUE DES DÉFAUTS D'HOMOGÉNÉITÉ}

Les modifications apportées à la lci d'approche à la saturation dépendent d'ailleurs de la nature des défauts qui les provoquent. C'est ainsi que l'effet des inclusions non magnétiques, des cavités ou des fluctuations de concentration dans les solutions solides, est surtout sensible dans les champs très élevés de l'ordre de plusieurs milliers de gauss; la loi d'approche prend d'ailleurs la forme approximative :

$$
\mathrm{I}=\mathrm{I}_{s}\left(1-\frac{a}{\mathrm{H}}\right)
$$

$\mathrm{Au}$ contraire, l'effet des tensions internes se manifeste surtout dans le domaine des champs moyens, entre 100 et 2.000 gauss, tandis que la loi d'approche prend la forme approchée :

$$
1=1_{b}\left(1-\frac{b}{\mathrm{H}^{2}}\right)
$$

nettement différente de la précédente. Il y a donc ici le principe d'une méthode de différenciation de ces deux catégories de défauts, fournissant en même temps une mesure de leur intensité. Elle pourrait trouver des app ications dans les études métallurgiques et fournir des résultats intéressants et utiles. On pourrait notamment étudier ainsi l'anisotropie produite par les traitements mécaniques, laminage, étirage, etc. Signalons à ce propos que la distinction qui vient d'être faite est quelquefois délicate : certains défauts produisent à la fois des tensions internes et l'effet d'inclusion : comme, par exemple, la ségrégation en deux phases d'une solution solide saturée.

\section{SENSIBILITÉ RELATIVE DIFFÉRENTE DU FER ET DU NICKEL AUX DIFFÉRENTS DÉFAUTS}

Le calcul montre d'ailleurs que la sensibilité relative aux deux catégories de défauts signalées plus haut varie énormément suivant la nature de la substance ferromagnétique : les unes sont très sensibles aux tensions internes comme le nickel et relativement insensibles à la présence des inclusions. Pour le fer, c'est exactement le contraire. C'est ainsi que la présence d'inclusions convenablement divisées, occupant dix pour cent du volume total, élève à 36 gauss le champ coercitif du fer et à 10 gauss seulement celui du nickel. Au contraire, des tension internes de $30 \mathrm{~kg} / \mathrm{mm}^{2}$, irrégulièrement réparties, n'élèvent que de 2 gauss le champ coercitif du fer tandis qu'elles amènent à 330 gauss celui du nickel. La raison profonde de cet état de choses tient à la grande magnétostriction du nickel accompagnée d'une petite anisotropie magnétocristalline et d'une aimantation à saturation relativement faible, tandis que dans le fer, l'anisotropie magnétocristalline et l'aimantation à saturation sont grandes et la magnétostriction petite. On commence donc à avoir des idées suffisamment précises sur l'origine exacte de l'hystérésis pour espérer en tirer parti. Le fait mérite d'être signalé car, d'après les théories de l'hystérésis magnétique encore en cours en 1939, on était obligé de conclure à la présence dans le fer doux recuit de tensions internes résiduelles de l'ordre de 5 à $10 \mathrm{~kg} / \mathrm{mm}^{2}$, ce qui n'aparaissait pas très vraisemblable; aujourd'hui on sait que des inclusions occupant quelques millièmes du volume total suffisent à interpréter les résultats de l'expérience.

On comprend aussi maintenant beaucoup mieux le rôle des traitements theimiques aux températures très élevées qui ont été reccnnus nécessaires pour améliorer encore les propriétés magnétiques des alliages hautement perméables : Bozcrth a montré, par exemple, qu'en traitant à $1.300^{\circ} \mathrm{C}$ par de l'hydrogène pur et sec un permalloy de composition appropriée, la perméabilité initiale passait de 10.000 à plus de 100.000 . On élimine ainsi les derrit̀̀rss traces d'inclusions aux limites des grains. 


\section{LES ALLIAGES POUR AIMANTS PERMANENTS}

Un autre exemple instructif est celui des alliages pour aimants permanents. La théorie indique que pour obtenir un champ coercitif aussi grand que possible il faut que la substance présente des tensions internes aussi grandes que possible, irrégulièrement distribuées, en même temps que des micro-inclusions occupant un grand volume relatif, l'échelle de ces défauts étant convenable, de l'ordre de $1 / 10^{\circ}$ à 1 micron. Les aimants en poudre de fer dont nous avons parlé plus haut rentrent, si l'on veut, dans ce type général : les procédés employés dans leur fabrication peuvent être considérés comme une méthode synthétique d'obtention de trous, mais les mêmes résultats s'obtiennent à peu près automatiquement dans les alliages à durcissement par dispersion dans lesquels une solution solide homogène à haute température se décompose par refroidissement en deux phases distinctes. Par exemple dans les nickel-béryllium, le béryllium, soluble à haute température, se sépare à basse température; on obtient donc des grains de béryllium isolés dans une matrice formée d'une solution de béryllium dans le nickel. Ces grains jouent le rôle d'inclusions en même temps qu'ils provoquent l'apparition de tensions internes. De même, dans les fer-nickel-aluminium voisins de $\mathrm{Fe}^{2} \mathrm{NiAl}$, la solution solide, formée à haute température des atomes distribués au hasard suivant les différents sites du réseau, se décompose à basse température en deux phases appartenant au même réseau cristallin, mais de composition et de paramètres très différents. L'une des phases, constituée de fer presque pur, est très magnétique; l'autre, qui contient presque tout le nickel et l'aluminium est beaucoup moins magnétigue et se comporte comme une inclusion vis-à-vis de la première. $\mathrm{Par}$ refroidissement rapide, on peut obtenir des grains très fins qu'une revenu ultérieur fait grossir : on peut donc donner aux défauts l'échelle la plus favorable au moyen d'un traitement thermique convenable.

Ces fer-nickel-aluminium, additionnés de cobalt, de vanadium, etc., constituent d'excellents aimants. Traités thermiquement dans un champ magnétique, ils deviennent anisotropes pour une certaine composition et présentent, dans la direction du champ magnétique qui a été appliqué pendant le refroidissement, une aimantation rémanente élevée. Ce phénomène est probablement dû à l'allongement des grains de l'une des phases sous l'influence du champ démagnétisant interne. Il sera d'autant plus aisé à produire que la tension interfaciale des deux phases sera plus faible et que le point de Curie sera plus élevé, ce qui permet d'opérer dans une zone de température suffsamment élevée pour que la recristallisation soit rapide. Il y a là le principe d'une méthode d'étude et de mesure des tensions interfaciales à l'état solide.

A cause de leur mode de préparation, tous ces alliages à aimants sont très durs et très difficiles à usiner. On peut également obtenir la dispersion d'inclusions à l'échelle désirée en s'adressant à des alliages tels que des fercobalt-vanadium, caractérisés par l'existence d'une certaine phase unique $\gamma$ stable à haute température, d'une autre phase unique $\alpha$ stable à basse température, ces deux domaines de température étant séparés par une région intermédiaire à l'intérieur de laquelle les deux phases $\alpha$ et $\gamma$ peuvent coexister en équilibre. Lorsqu'on fait subir à cette substance un refroidissement rapide, cette région intermédiaire est traversée assez vite pour que la ségrégation en deux phases, dont les compositions sont d'ailleurs légèrement différentes, ne puisse pas s'effectuer car les atomes des différents constituants n'ont pas le temps matériel de diffuser à longue distance : il en résulte qu'à basse température on obtient la phase $\alpha$ à l'état pur. Corrélativement, la dureté mécanique est assez faible pour que l'alliage soit facile à usiner. $\mathrm{Si}$ alors on réchauffe la subştance, de manière à la ramener dans la zone intermédiaire, en la laissant séjourner suffisamment longtemps à cette température, les phases se séparent en grains distincts qu'il est possible ensuite de conserver en faux équilibre à la température ordinaire par refroidissement rapide. Des alliages de ce type, contenant $50 \%$ de cobalt et $10 \%$ de vanadium mis au point par Nesbitt sont actuellement employés aux Etats-Unis sous le nom de vicalloys.

\section{CONCLUSION}

Les quelques exemples, choisis parmi beaucoup d'autres, que je viens de citer, montrent suffisamment limportance et la nature des renseignements que peut fournir l'étude des propriétés magnétiques d'un alliage dont l'une au moins des phases est ferromagnétique. En ce qui concerne les applications pratiques des alliages magnétiques, il ne faut pas cependant s'exagérer l'importance des théories plus approfondies et plus rigoureuses de l'hystérésis, telles qu'on les développe actuellement. Fort heureusement, pour fabriquer des aimants, ou bien des tôles de transformateur et de dynamo avec des pertes raisonnablement faibles, il n'a pas fallu attendre de comprendre le mécanisme de l'aimantation et d'en développer la théo- rie. Mais il faut dire aussi que les efforts qui ont été déployés dans l'étude empirique des phénomènes ont été incomparablement plus grands que ceux qui ont été consacrés à leur interprétation. Dans un domaine aussi complexe que celui de la métallurgie, il paraît donc raisonnable d'attendre au moins, d'une meilleure compréhension des phénomènes, une économie notable de temps et de maind'œuvre : ne serait-elle que de $20 \%$, ce serait encore important. Naturellement, je ne compte que pour mémoire, car elle ne figure pas dans les bilans, la satisfaction profonde qu'éprouve le physicien à mieux comprendre les phénomènes qui font l'objet de ses recherches. 